\title{
The possibility of using a smartphone in a single plane rotor balancing
}

\author{
A. AIT BEN AHMED ${ }^{1}$, A. TOUACHE ${ }^{2}$, A. EL HAKIMI ${ }^{3}$, A. CHAMAT ${ }^{4}$
}

1. Laboratory of Mechanical Engineering, FST Fez, abdelbaset.aitbenahmed@ gmail.com

2. Laboratory of Mechanical Engineering, FST Fez, abdelhamid.touache@gmail.com

3. Laboratory of Mechanical Engineering, FST Fez, abdelhadi.elhakimi@ usmba.ac.ma

4. Laboratory of Mechanical Engineering, FST Fez, abderrahim.chamat@ usmba.ac.ma

\begin{abstract}
This paper aims to investigate and to describe the possibility of using the smartphones in the rotors balancing. It shows in the beginning a method to extract the necessary parameters for implementing the field balancing operation. An Android app was developed in order to record the smartphone sensors data of unbalanced rotors. The recorded data is then processed and used to identify the quantity of mass necessary and its position via a Matlab app. At the end, the smartphone results were compared by the exact results.
\end{abstract}

Keywords: Smartphone, balancing rotor, signal processing, Vibration Monitoring.

\section{Introduction}

A bad distribution of mass in a rotor will lead to the appearance of the unbalance defect, which causes an undesirable vibration of the rotor [1], this defect necessitates necessarily to correct it. This correction is called balancing. So, the balancing is a process that allows us to control the distribution of masses in a rotor and improve it to maintain within acceptable limits the forces and vibrations caused by unbalance [2]. The balancing operation is very important for improving the reliability of a machine and extending its life. The balancing procedure by professional equipment is considered expensive, although inevitable; this leads us to seek to minimize the costs of this operation and to be more applicable for professionals and non-professionals.

In this stage we deal primarily with the balancing of rigid rotors, which are characterized by operation in the subcritical domain [3]. The balancing studied in this work is called field balancing, which requires only a vibration measuring device that can measure the amplitude and phase of the vibration signal.

In recent years, smartphones have found their place in our daily lives. They are always with us and we can use them simply and quickly. In this field we have exploited the smartphones to use them in the balancing operation.

This paper will devote to investigate and to describe how to use the smartphones in the balancing procedure.

In this work we used the smartphone as a device for the acquisition of rotor vibration via an application developed by us, called "vibroteak" which was developed on the most widespread operating system; "Android" [4], using this application we were able to record the vibration signals generated by unbalanced rotors.

After the acquisition step, we processed the record data via a dedicated Matlab application for the treatment of machine defects. In the end, we compared the smartphone results by the theoretical results.

\section{The data acquisition}

The smartphone that we used in this work has a built-in MEMS (Micro Electro Mechanical Systems) type accelerometer, manufactured by the Bosch Company with the following features. (Fig.1)

\section{Sensor Info \\ Accelerometer: Detected \\ Name $=$ BOSCH BMC150 Acceleration Sensor \\ Vendor $=$ Bosch Sensortec, $\mathrm{GmbH}$ \\ Version $=1$ \\ Resolution $=0.009575196$ \\ Max Range $=156.88$ \\ Power $=0.13 \mathrm{~mW}$ \\ Fig. 1: Accelerometer features.}

Through this accelerometer, we can record vibrations up to $156 \mathrm{~m} / \mathrm{s}^{2}( \pm 16 \mathrm{~g})$ with a resolution of $0.0095 \mathrm{~m} / \mathrm{s}^{2}$ (the smallest increment or decrement vibration value detected by the sensor), consuming 0.13 $\mathrm{mW}$ of power.

Regarding the sampling frequency; the accelerometer was sampled regularly due to the implementation of the sampling mechanism by the Android structure. The Android API offers four abstract 
sample rates for its accelerometer sensor (listed from the fastest to the slowest): Fastest, Game, Normal, and UI [5]. In this work we chose the fastest mode; Fastest mode has given us about $100 \mathrm{~Hz}$ as a sample frequency.

The coordinate system is set relative to the phone screen in its default orientation, the axes are not exchanged when the screen orientation of the device changes. The $\mathrm{X}$ axis is horizontal, the $\mathrm{Y}$ axis is vertical, and the $\mathrm{Z}$ axis is directed to the outside of the front face of the screen. (Fig.2)

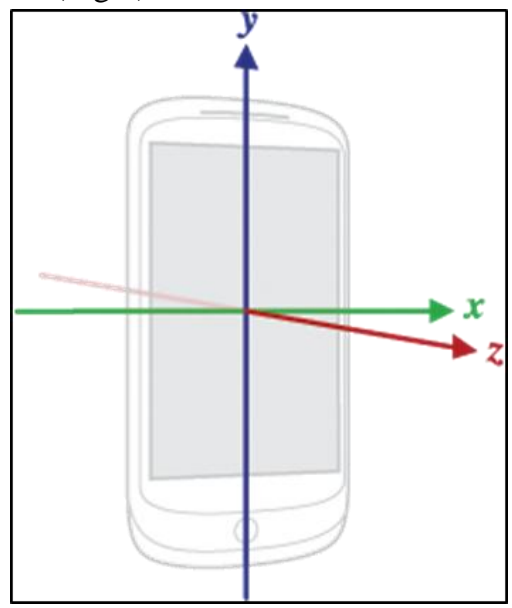

Fig. 2: The coordinate system of sensors in a smartphone. [6]

The application "vibroteak" allowed us to record the vibration data via the accelerometer built into the smartphone and store it in a file (.CSV), and also allowed us to record the reference signal using the microphone, under the extension (.wav). The recording of the both signals (vibration and reference) is done simultaneously.

\subsection{The amplitude measurement}

For amplitude acquisition we need to fix the smartphone on the bearing as shown in the figure 4 .

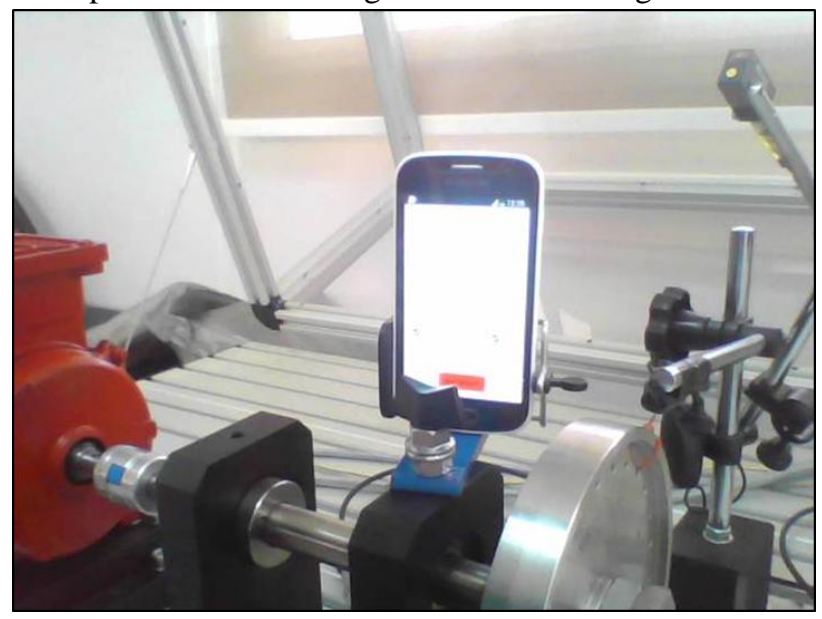

Fig. 4: the smartphone fixed on the bearing.

We set the machine to $1500 \mathrm{rpm}$, because of the existing unbalance, the rotor generates a vibration along the rotor and especially on the bearings. The smartphone records the vibration signal in three dimensions $(\mathrm{X}, \mathrm{Y}$, $\mathrm{Z}$ ), with the maximum acquisition frequency ( $\mathrm{Fs}=100$
$\mathrm{Hz}$ ). After a 15 seconds of acquisition, we stopped the record and saved the data.

\subsection{Phase measurement}

For phase acquisition, we need a reference signal, for that, we have proposed an acoustic method to build it. Our method is based on the using the microphone of the smartphone as a tachometer (Fig. 5). We used a mark to create a sound pulse when the disk completes a tower (Fig. 6), after the processing of the sound signal recorded by the microphone; we obtained the reference signal. (Fig. 7) and (Fig. 8)

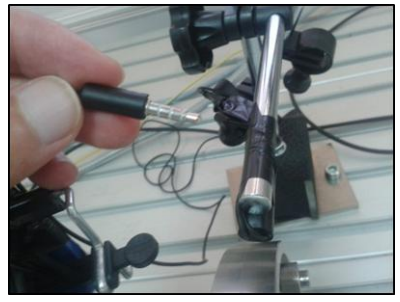

Fig. 5: the microphone connected to the Smartphone.

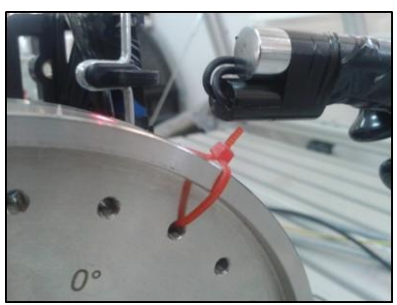

Fig. 6: Sound pulse creation via the red mark.

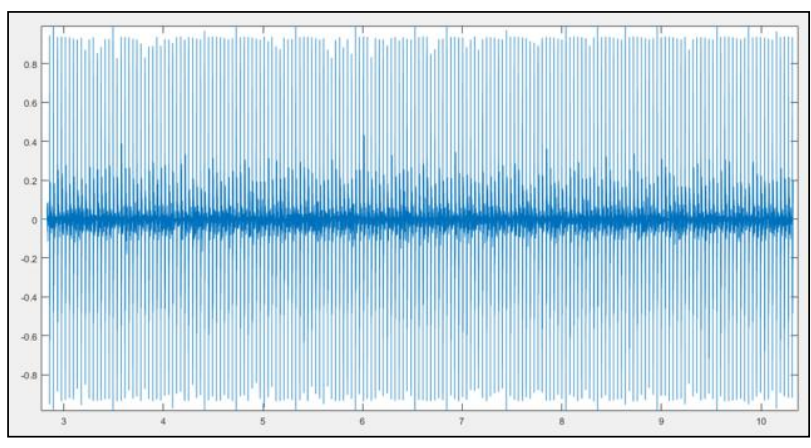

Fig. 7: sound wave before processing at $1500 \mathrm{rpm}$

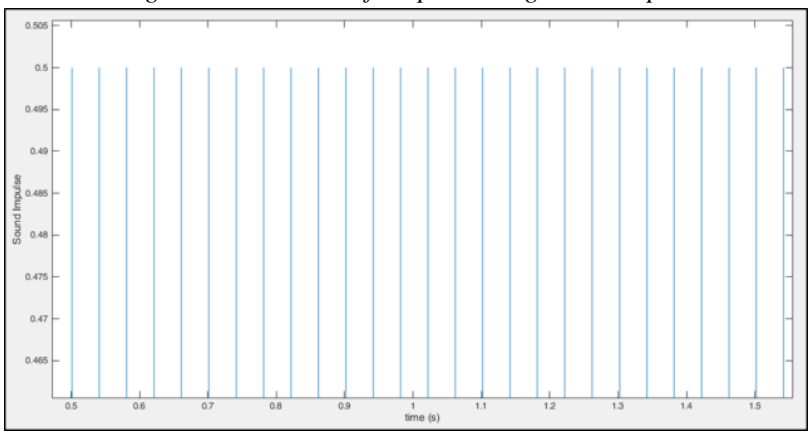

Fig. 8: the reference signal obtained after processing at $1500 \mathrm{rpm}$.

\section{Influence coefficient balancing method}

The influence coefficient method is the most usable method for balancing dynamic rotors by maintenance personal [7], [8]. It has the objective to eliminate the vibration at a number of measuring points for one or more rotor operating speeds. This method is based on the assumption of the linearity of the system [9].

$$
\{V\}=[C] *\{B\} \quad(1)
$$

$\{\mathrm{V}\}$ : Vector vibrations of the rotor in several planes. 
$\{B\}$ : Vector unbalance in the rotor.

[C]: Matrix of influence coefficients.

Equation (1) reflects the linearity of the system in the general case, in the case of a single balancing plane, equation (1) becomes:

$$
V=C^{*} B
$$

$\mathrm{V}$ : Vibration of the rotor in one plane.

$B$ : The unbalance in the rotor.

C: The influence coefficient.

This method consists in calculating the coefficient of influence $\mathrm{C}$ from the test weight $\mathrm{P}$, and the calibration vector $\mathrm{T}$ (the vector obtained when we add the trial mass), by the relation (3):

$$
C=P / T
$$

\section{Results and discussion}

As shown in the table 1, we made four tests with different weights and at different positions. This table summarize the results obtained by the smartphone in each test; compared with the exact results.

Table 1: rotor balance results at $1500 \mathrm{rpm}$

\begin{tabular}{|c|c|c|c|c|}
\hline $\begin{array}{c}\text { Test } \\
\mathbf{N}^{\circ}\end{array}$ & $\begin{array}{c}\text { Original } \\
\text { unbalanc } \\
\left(\mathrm{g} @ 0^{\circ}\right)\end{array}$ & $\begin{array}{c}\text { Trial weight } \\
\left(\mathrm{g} @ 0^{\circ}\right)\end{array}$ & $\begin{array}{c}\text { Experimental } \\
\text { Results } \\
\left(\mathbf{g} @{ }^{\circ}\right) \\
{[\mathrm{by} \text { smartphone }}\end{array}$ & $\begin{array}{c}\text { Exact } \\
\text { results } \\
\left(\mathrm{g} @{ }^{\circ}\right)\end{array}$ \\
\hline 1 & $10.7 \mathrm{~g} @ 225^{\circ}$ & $10 \mathrm{~g} @ 315^{\circ}$ & $10.11 \mathrm{~g} @ 51^{\circ}$ & $10.7 \mathrm{~g} @ 45^{\circ}$ \\
\hline 2 & $10.7 \mathrm{~g} @ 225^{\circ}$ & $10 \mathrm{~g} @ 60^{\circ}$ & $9.98 \mathrm{~g} @ 82.12^{\circ}$ & $10.7 \mathrm{~g} @ 45^{\circ}$ \\
\hline 3 & $12 \mathrm{~g} @ 180^{\circ}$ & $15 \mathrm{~g} @ 270^{\circ}$ & $14.30 \mathrm{~g} @ 1.28^{\circ}$ & $12 \mathrm{~g} @ 0^{\circ}$ \\
\hline 4 & $15 \mathrm{~g} @ 270^{\circ}$ & $15 \mathrm{~g} @ 315^{\circ}$ & $16.39 \mathrm{~g} @ 145^{\circ}$ & $15 \mathrm{~g} @ 90^{\circ}$ \\
\hline
\end{tabular}

Figure 9 shows the 1 plane balancing interface of Matlab application. (Test $\left.\mathrm{N}^{\circ} 1\right)$

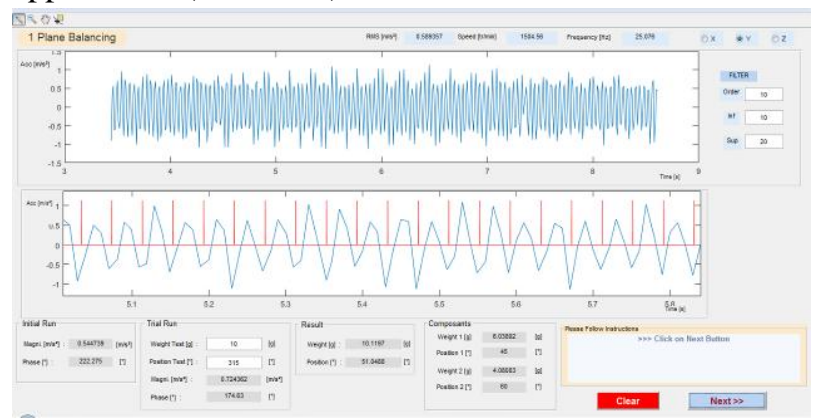

Fig.9 One Plane balancing interface $\left(\right.$ Test $\left.N^{\circ} 1\right)$

At the first, the exact results was calculated supposing the used rotor is perfectly balanced. So, The deference between the results obtained by the smartphone and the exact results can be justified by the sampling frequency of the smartphone (Fs), which the big constraint that we faced in this work, for overcoming this problem, whether increase the sampling frequency or decrease the speed of rotor. The remarkable instability of the phase lead us to start a study on the change of phase according to trial weight position in the future work.

\section{Conclusions}

The application "Vibroteak" allows to measure the parameters necessary for the balancing procedure. As we saw in the Table 1, the smartphone gives an acceptable results, which push us to continuing this investigation to building a framework based on the smartphone to control the unbalance defects.

\section{References}

[1] ISO 1940/1 - Mechanical vibration - Balance quality requirements for rotors in a constant (rigid) state - Part 1: Specification and verification of balance tolerances, vol. 2003, p. 36, 2003.

[2] H. Schneider, Technique d'équilibrage, Springer, Paris, 2003.

[3] Balance Quality Requirements of Rigid Rotors. The Practical Application of ISO 1940/1, Quality, p. 11, 2009.

[4] Google, Sensor | Android Developers, [Online]. Available: https://developer.android.com/reference/android/hardware /Sensor. [Accessed: 29-Oct-2018].

[5] Xamarin, SensorDelay Enumeration [Online], Available: https://developer.xamarin.com/api/type/Android.Hardware .SensorDelay/. [Accessed: 31-Oct-2018].

[6] Google, SensorEvent | Android Developers, [Online]. Available:https://developer.android.com/reference/android /hardware/SensorEvent\#values. [Accessed: 29-Oct-2018].

[7] R. Kelm, D. Pavelek, and W. Kelm, Rotor Balancing Tutorial, 45th Turbomacninery 32nd Pump Symp, 2016.

[8] W. C. Foiles, P. E. Allaire, and E. J. Gunter, Review: Rotor balancing, Shock Vib., vol. 5, no. 5-6, pp. 325-336, 1998.

[9] C. Alauze, Equilibrage actif des machines tournates: Application aux grandes lignes d'arbres, INSA Lyon, 1998. 